\title{
Foams in a rotating drum: An analogy with granular materials
}

\author{
A. Bronfort*, H. Caps \\ GRASP, Physics Department B5, University of Liège, B-4000 Liège, Belgium
}

\section{H I G H L I G H T S}

- We compare the experimental results for granular matter and for foams.

- The drum rotation rises the foam liquid fraction and deforms the interface.

- A dimensionless viscous number similar to those for grains is defined

- A dilatancy law is modeled.

- A effective friction is defined and modeled with a law similar to granular friction.

\section{A R T I C L E I N F O}

\section{Article history:}

Received 30 September 2014

Received in revised form 5 January 2015

Accepted 6 January 2015

Available online 13 January 2015

\section{PACS:}

45.70.Qj

47.15.gp

47.35.-i

\section{Keywords:}

Hele-Shaw flows

Foam flows

Granular matter

\section{G R A P H I C A L A B S T R A C T}
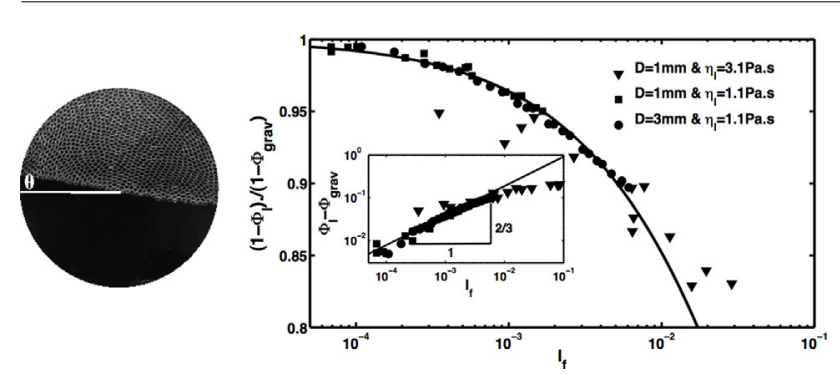

\begin{abstract}
A B S T R A C T
Rotating drums are usually used to study the flowing properties of granular materials. Here, we report experiments performed in a drum made of a circular Hele-Shaw cell filled with a monodisperse foam and its surfactant solution. The cell is placed vertically and rotated around its center with an angular velocity $\omega$. The rotation of the cell modifies the foam properties and therefore influences its response to the external strain. The observed foam behavior is similar to granular media in several aspects. Using tools developed for granular materials, we characterize the foam evolution in terms of a dimensionless viscous number $I_{f}$ and we compare the foam rheology to the theory developed for the immersed granular material rheology.
\end{abstract}

(C) 2015 Elsevier B.V. All rights reserved.

\section{Introduction}

Granular materials are dense assemblies of small solid particles separated by an interstitial fluid. They are encountered in numerous industrial applications, such as construction materials and pharmaceuticals [1,2]. Therefore granular flows have been extensively studied in various geometries [3-5]. One of them might be the rotating drum half-filled with grains. In this geometry, the granular flow might be divided into 2 areas. The first one is along the

\footnotetext{
* Corresponding author. Tel.: +32 43664831.

E-mail address: abronfort@ulg.ac.be (A. Bronfort).
}

outer wall of the drum where the grains constitute a solid rotating with the angular velocity of the drum. The second area is a flowing layer at the surface of the grain assembly. Depending on the drum angular velocity, several flow regimes occur at the surface. For example, at relatively low angular velocities, the surface inclines and the grains flow along a flat interface, while at large angular velocities, the inclined surface is deformed into an S-shape $[6,7]$. These phenomena have essentially been studied for dry granular media. Recently interest has been shown in wet and immersed granular flows and in the effect of the interstitial fluid on the flow $[8,9]$.

In the last few years, efforts have been made to unify the rheology of dry and immersed granular materials [7,10-12]. Dry 


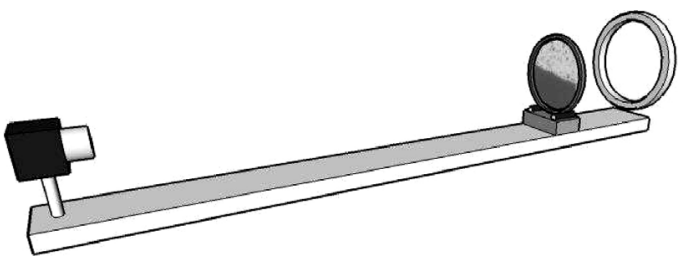

Fig. 1. Sketch of the experimental setup.

granular flows can be phenomenologically described by a frictional rheology based on only one dimensionless control parameter, the inertial number $I$ [3]. This number is interpreted as the ratio between the time of rearrangement and the mean time spent by a grain to move from one hole to the next one. Any dry granular material sheared at a given shear rate $\dot{\gamma}$ under a confining pressure $P_{p}$ is fully characterized by two constitutive laws: a friction law $\tau=\mu(I) P_{p}$ and a particle volume fraction law $\phi=\phi(I)$ with $\tau$ the shear stress, $\mu(I)$ the effective friction coefficient $[13,14]$. For immersed granular materials, also called granular suspensions, this formalism can be applied providing the assumption that the interstitial fluid between the suspended particles only influences the time scale of their relative motion. A modification of the dimensionless control number $I$ is then required and should account for the properties of the particle assembly as well as of the interstitial fluid [15].

In the literature, foams have since often been compared to granular materials [16-18]: they are composed of small immersed particles and their flow properties are similar to that of classical granular flows. In this paper, we propose to study the flow, in a narrow rotating drum, of monodisperse foams immersed in their solution. We propose to compare the foam rheology and the granular rheology $[10,15]$ adapting the dimensionless number I and using the constitutive laws developed for granular materials.

\section{Experimental setup}

The experimental setup consists of a circular Hele-Shaw (HS) cell made of polycarbonate transparent plates with a radius $R=65 \mathrm{~mm}$ and a thickness $e=3 \mathrm{~mm}$. The bottom half of the cell is filled with an aqueous surfactant solution while the other half is filled with a monodisperse foam made with a milli-fluidic Tjunction [19]. Afterwards the filled cell is placed vertically and rotated around its center with only one control parameter, the cell angular velocity $\omega$, ranging in $[0 ; 2.55] \mathrm{rad} / \mathrm{s}$. The setup is backlighted and the images of the foam are recorded using a high-speed video camera with a frame rate of $100 \mathrm{fps}$ (Fig. 1).

Three different foams are tested. They are made of an aqueous solution composed of $1 \%$ of a commercial dishwashing soap and of a few percents of glycerol. The gas used in the three cases is ambient air. The bubble size as well as the glycerol percentage of the solution are varied. The characteristics of the three cases are summarized in Table 1.

Before each experiment the foam is left at rest for a few minutes in order to reach an equilibrium state. Due to the gravity, the

Table 1

Characteristics of the three different foams. $D$ is the bubble diameter, $\eta_{l}$ the dynamic viscosity of the solution, $\rho_{l}$ the solution density, $\gamma$ the solution surface tension and $\phi_{\text {grav }}$ the foam mean liquid fraction at hydrostatic equilibrium.

\begin{tabular}{llll}
\hline & Case a & Case b & Case c \\
\hline$D(\mathrm{~mm})$ & $1 \pm 4 \%$ & $3 \pm 2 \%$ & $1 \pm 4 \%$ \\
Glycerine $(\%)$ & 5 & 5 & 30 \\
$\eta_{l}(\mathrm{mPa} \mathrm{s})$ & 1.1 & 1.1 & 3.1 \\
$\rho_{l}\left(\mathrm{~kg} / \mathrm{m}^{3}\right)$ & 1008 & 1008 & 1087 \\
$\gamma(\mathrm{mN} / \mathrm{m})$ & 25 & 25 & 25 \\
$\phi_{\text {grav }}$ & 0.025 & 0.01 & 0.024 \\
\hline
\end{tabular}

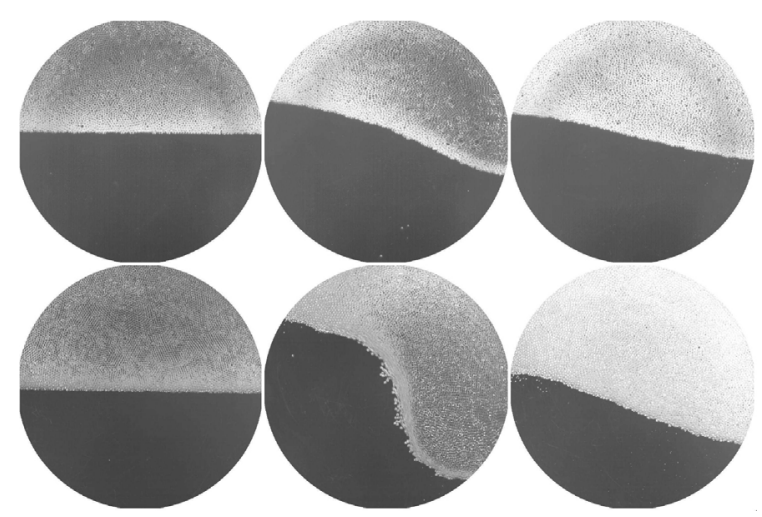

Fig. 2. Pictures of the half-filled HS cells. The rotation direction is clockwise. The first row corresponds to an angular velocity $\omega=0.85 \mathrm{rad} / \mathrm{s}$ while the second one corresponds to $\omega=2.55 \mathrm{rad} / \mathrm{s}$. The first column shows the foam at rest, the second one the foam a few seconds after the rotation begins and the third one the foam after more than $15 \mathrm{~s}$. Case a.

liquid inside the Plateau borders of the foam flows downwards until the liquid weight is balanced by the capillary forces. The flow then becomes negligible and the foam has reached the so-called hydrostatic equilibrium. The foam liquid fraction is then ruled by the equation [20]:

$\phi_{l}(y)^{-1 / 2}-\phi_{l}^{*-1 / 2} \simeq \frac{y D}{l_{c}^{2}}$,

with $y$ the vertical position with its origin at the foam/liquid interface, $D$ the bubble diameter and $l_{c}$ the capillary length. Considering $\phi_{l}^{*}=0.26$, the liquid fraction for an ordered 3D spherical bubble foam, this equation allows us to compute the foam mean liquid fraction $\phi_{\text {grav }}$ at hydrostatic equilibrium of a half-filled cell. The results for the three considered foams are represented in Table 1.

\section{Foam dynamics}

As the HS cell is set in motion, the bubbles start to flow along the foam/liquid interface and follow a circular trajectory in the same direction than the cell but with a slightly slower velocity. The bubbles rising from the interface bring liquid to the foam increasing its mean liquid fraction. The foam interface is also modified by the cell rotation. It inclines and deforms into a S-shape. Fig. 2 presents three states of a foam in rotation for two different cell angular velocities. The first column corresponds to two foams at rest at hydrostatic equilibrium. The second column represents the same foams a few seconds after the rotation begins. The third column presents them long after the beginning of the experiment, when they have reached their dynamic equilibrium. These images evidence the rise in liquid fraction through the variations of the foam brightness: the brightest the foam, the highest the liquid fraction. This figure also highlights the deformation of the interface. The amplitude of the deformation is the largest a few seconds after the beginning of the experiment. Afterwards it decreases to reach a stable equilibrium position. The amplitude of the deformation grows with the angular velocity $\omega$. The deformation of the interface is divided into two phenomena: the growth of the deformation and its attenuation. The increase of the foam liquid fraction seems to match the attenuation of the interface deformation.

The two phenomena of the interface deformation have been represented on two distinct figures. Fig. 3 presents the growth of the interface deformation, from its position in the hydrostatic equilibrium state to its maximal deformation. The first solid line is plane and horizontal which corresponds to a foam at rest. As the cell starts rotating, the interface inclines while remaining plane and the foam rotates like a solid. This state is represented by the 


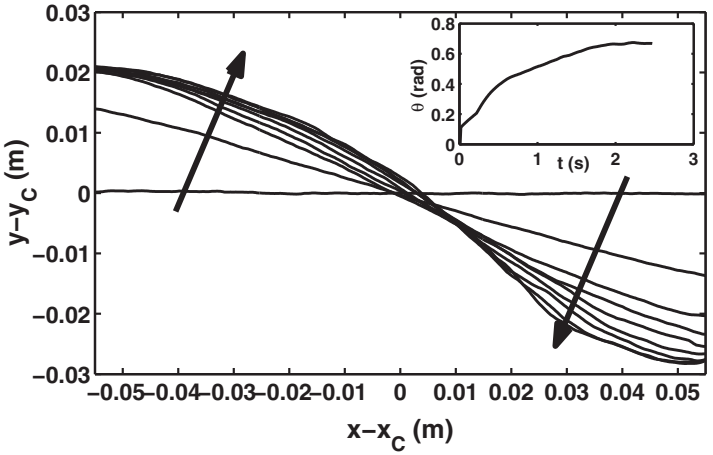

Fig. 3. Interface deformations from rest to its maximal deformation. The curves are centered on the cell center $\left(x_{C} ; y_{C}\right)$ and the arrows indicate the moving direction of the interface and so the time evolution. Curves are apart from $\Delta t=0.25 \mathrm{~s}$ during approximately $2.4 \mathrm{~s}$. The inset is the angle $\theta$ of the interface with the horizontal at the inflection point versus the time. Case a with $\omega=1.19 \mathrm{rad} / \mathrm{s}$.

second solid line. Afterwards the foam starts flowing clockwise, the interface deforms into a S-shape with an increasing inclination angle $\theta$ which is defined as the angle between the interface at its inflection point and the horizontal. The time evolution of $\theta$ is presented in the inset. The interface on the rising side stabilizes quickly while on the downward side the interface deformation continues to increase a bit longer. This results in a vertical asymmetry between both sides. After a few seconds the deformation is maximal and begins to reduce. Fig. 4 represents the attenuation of the interface deformation, from its maximal amplitude to its shape in the dynamic equilibrium state. As the interface is about to reach the dynamic equilibrium position, the deformation variation slows down. At the dynamic equilibrium the interface keeps a S-shape and its inclination angle is non-null. The vertical asymmetry also seems to have lessened.

The observed foam/liquid interface dynamics is reminiscent of the behavior of granular materials. In a narrow drum filled with grains, as the rotation of the cell begins, the interface inclines while the assembly of grains moves like a solid. As a critical inclination angle is reached, the grains starts to flow along the interface [21-23]. At large angular velocity values, the grain interface (dry or immersed) exhibits a S-shape. As observed with foams, the deformations of the granular assembly interface depend on the cell angular velocity $\omega$. The inclination angle as well as the S-shape amplitude increase with $\omega[7,24,25]$. These observations clearly evidence the similarities between foams and granular materials.

To understand the physical mechanisms generating the deformation of the foam interface, it is essential to estimate the

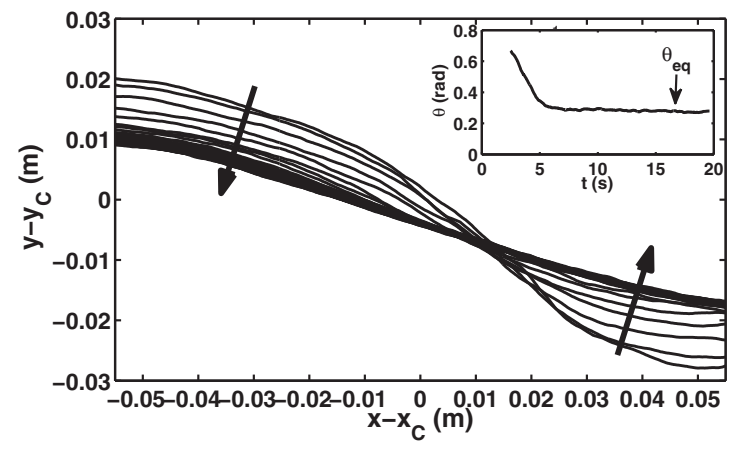

Fig. 4. Interface deformations from its maximal deformation to its dynamical equilibrium. The curves are centered on the cell center $\left(x_{C} ; y_{C}\right)$ and the arrows indicate the moving direction of the interface. $\Delta t=0.5 \mathrm{~s}$ during approximately $17 \mathrm{~s}$. The inset is the angle $\theta$ of the interface with the horizontal at the inflection point versus the time. Case a with $\omega=1.19 \mathrm{rad} / \mathrm{s}$.

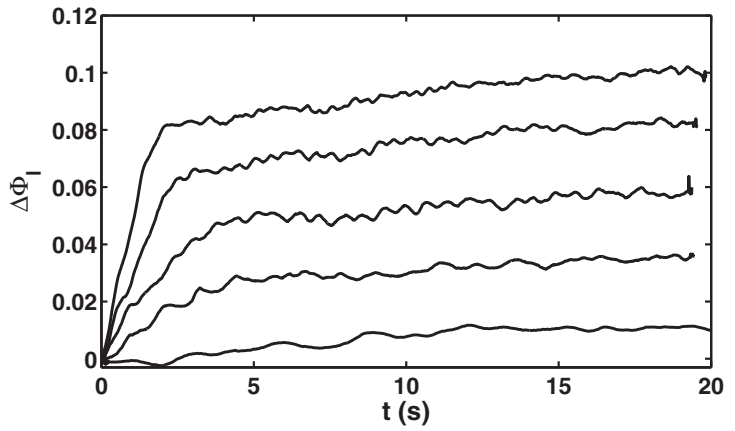

Fig. 5. Variations with time of the foam liquid fraction $\Delta \phi_{l}$ for different cell angular velocities $(\omega=0.25 \mathrm{rad} / \mathrm{s}, \omega=0.68 \mathrm{rad} / \mathrm{s}, \omega=1.1 \mathrm{rad} / \mathrm{s}, \omega=1.61 \mathrm{rad} / \mathrm{s}, \omega=1.95 \mathrm{rad} / \mathrm{s})$. Case a. $\Delta \phi_{l}$ goes up with the angular velocity. For $t=0 \mathrm{~s}$, the cell is at rest and the foam liquid fraction is at the gravitational equilibrium.

variations of the foam properties due to the cell rotation. Among them, the most important one is the foam mean liquid fraction which influences the properties of the foam such as its viscosity.

A good experimental estimation of the variations of the foam liquid fraction can easily be obtained from the foam volume variations. As the gas might be considered as incompressible, the foam volume variations can only be attributed to a modification of the foam liquid fraction. Fig. 5 represents the temporal variations of the liquid fraction $\Delta \phi_{l}(t)$ between the foam at rest and the same foam rotated for a time $t$. Since experiments start with a foam at rest, the first value for all the curves is null. As soon as the cell is set in motion, all curves rapidly increase implying that a large amount of liquid is injected in the foam per unit of time. After a while the rise in liquid fraction slows down significantly. Fig. 5 also evidences the link between $\Delta \phi_{l}(t)$ and the cell angular velocity $\omega$. The larger the velocity, the larger the liquid fraction variation.

Fig. 5 points out two different regimes. The first one is the fast increase in liquid fraction mainly due to the viscous entrainment of liquid out of the bath by the walls of the cell. Indeed a plate pulled out of a wetting bath entrains a liquid film with a constant thickness that depends on the plate velocity [26,27]. In the second part of the curves, the liquid fraction increases more slowly. This is due to the competition between the capillary forces adding liquid to the layers at the interface and the gravitational drainage of the upper layers of the foam. When the equilibrium between these forces is reached, the foam liquid fraction is almost constant. Comparing Figs. 3-5 emphasize that the end of the first regime of the liquid fraction corresponds approximately to the end of the deformation growth. Moreover, at a given cell velocity $\omega$, as the liquid fraction rises, the interface deformation reduces. Both phenomena reaches the dynamic equilibrium approximately at the same time.

From this point of view the liquid fraction seems to play a major role in the dynamics of the foam/liquid interface. Fig. 6 presents the foam mean liquid fractions for the three different foams $a, b$ and $c$ as a function of the cell angular velocity $\omega$. Those mean liquid fractions $\phi_{l}$ are obtained by adding the mean liquid fraction at hydrostatic equilibrium $\phi_{\text {grav }}$ to the liquid fraction variation $\Delta \phi_{l}$. For all three cases, $\phi_{l}$ increases linearly with the cell velocity but the slope of the curve depends on the foam properties such as the bubble diameter: the larger $D$, the smaller the liquid fraction. The solution viscosity also plays a major role. The liquid fraction in the case $c$ is significantly larger than for the two other cases. This figure shows values of the liquid fraction larger than the critical liquid fraction of a spherical bubble foam $\phi_{l}^{*}$. The liquid film entrained by the cell walls is responsible for this result.

The foam mean liquid fraction affects the foam rigidity, i.e. its resistance to an external strain, by tuning the effects of the cohesion between bubbles. As the liquid fraction increases, the Plateau 
borders thicken and the bubbles becomes more and more spherical. The foam losses its rigidity and flows more easily under external strains. The foam effective viscosity gives a good quantification of this rigidity and is defined by the ratio of the external strain over the strain rate:

$\eta_{\text {eff }}=\frac{\sigma}{\dot{\epsilon}}$.

This effective viscosity depends on the external strain and on the properties of the foam. In our experiments, the foam might be considered incompressible. Moreover, the range of strain rate studied here is way above the quasi-static rate. Therefore only the shear viscous strain is relevant to compute the effective viscosity. It is given by the law developed by Tcholakova et al. [28,29] and results in the following viscosity:

$\eta_{\text {eff }}=\left(\frac{\mathrm{Ca}^{-0.535}}{2 \sqrt{\phi_{l}}}+\frac{6.2 \mathrm{Ca}^{-0.3}}{\phi_{l}^{0.2}}\right)\left(1-\phi_{l}\right)^{5 / 6} \eta_{l}$.

where the capillary number $\mathrm{Ca}=\eta_{l} \dot{\epsilon} D / 2 \gamma$ with $\dot{\epsilon}$ the shear strain rate. In our experiments, the hypothesis of continuous medium and of affine velocity field are verified and the shear strain rate is given by the symmetric part of the velocity gradient [30]. The velocity gradient of the bubbles have been computed from the displacements of their center with a procedure similar to the one proposed in [31]. The resulting effective viscosity $\eta_{\text {eff }}$ of a foam in a rotating drum is presented in Fig. 7 as a function of the cell velocity [32]. It was computed for a foam with the properties of the case $b$ in a cell exactly the same as the one used here. Large $\eta_{\text {eff }}$ values are obtained for low velocity where the foam flows more like a solid. As $\omega$ increases, the viscosity decreases and tends to a constant value for high velocities values. Then the foam flows like a viscous fluid and relaxes more easily under an external strain.

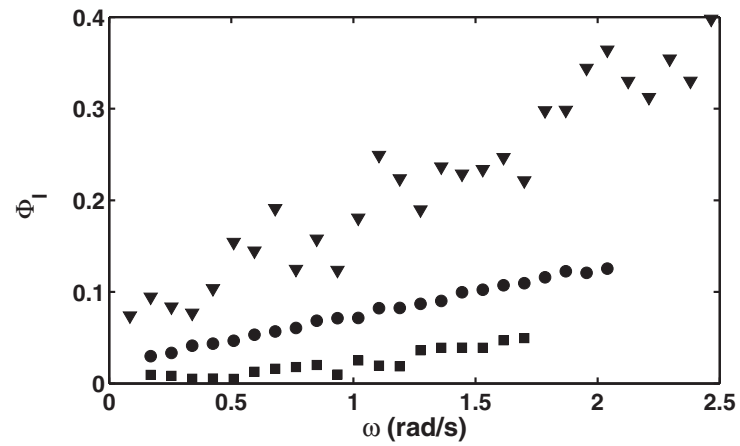

Fig. 6. The foam mean liquid fraction $\phi_{l}$ at the dynamic equilibrium versus the cell angular velocity $\omega$. Case a, $D=1 \mathrm{~mm}$ and $\eta_{l}=1.1 \times 10^{-3} \mathrm{~Pa}$. Case b, $D=3 \mathrm{~mm}$ and $\eta_{l}=1.1 \times 10^{-3}$ Pa s. $\mathbf{v}$ Case c, $D=1 \mathrm{~mm}$ and $\eta_{l}=3.1 \times 10^{-3}$ Pa s.

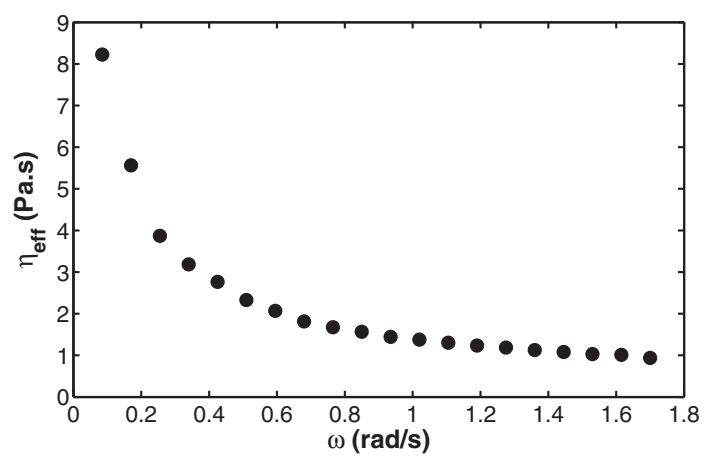

Fig. 7. Mean effective viscosity $\eta_{\text {eff }}$ of a type $\mathrm{b}$ foam as a function of the cell angular velocity $\omega[32]$.
The intrinsic properties of the foam are modified by the cell rotation. In its dynamic equilibrium, the foam liquid fraction, as well as its effective viscosity, depend strongly on the cell angular velocity. The observed behaviors of the present foams are reminiscent of the granular dynamics in rotating drums. On a microscopic level, foams and grain assemblies are very different from each other. However, on a macroscopic level, the comparison might be possible. The following section pushes further the macroscopic comparison between the granular rheology and the foam rheology applied to our specific results.

\section{Comparison with immersed granular materials}

The recent achievement in granular rheology led to a continuous description of granular flows based on the dimensionless control parameter $I[3,33,34]$. This number might be understood in terms of the characteristic times of the flow. During a simple shear at constant shear rate the first characteristic time $t_{p}$ is the mean time spent by a particle to move from one hole to the next one. In the present case, it is equal to the ratio between the bubble diameter $D$ and a characteristic bubble velocity. The foam characteristic velocity is half the velocity of the bubbles in contact with the outer wall of the cell. In our experimental set up, the outer wall is not textured and a slip is observed between the outer wall and the first layer of bubbles with a ratio 0.8 . The characteristic time $t_{p}$ is then equal to: $t_{p}=2 D / 0.8 \omega R$. The second one is the rearrangement time corresponding to the rapid fall of the particle from the unstable position at the top of the particle just below into the next hole. In the particular case of our foam, it reads: $t_{r}=\eta_{f} / P_{p}$ [15]. The confining pressure $P_{p}$ pushing the neighboring bubbles against each others is the osmotic pressure $\Pi[35,36]$. For a quasi two-dimensional monodisperse foam, the osmotic pressure is given by the following equation [37-39]:

$\Pi=\frac{2 \gamma}{D} \sqrt{\frac{1-\phi_{l}}{1-\phi_{l}^{*}}}\left[\sqrt{\frac{\phi_{l}^{*}}{\phi_{l}}}-1\right]$.

Eventually the foam dimensionless viscous number $I_{f}$ is defined as the ratio between the rapid rearrangement time $t_{r}$ and the mean time $t_{p}[3,15]$. It reads:

$I_{f}=\frac{t_{r}}{t_{p}}=\frac{0.8 \eta_{l} \omega R}{2 D \Pi}$.

This viscous number depends on the cell velocity and on the interstitial fluid properties while it depends on the bubble size only through the foam liquid fraction.

In order to validate this foam viscous number $I_{f}$, the foam bubble volume fraction $\left(1-\phi_{l}\right)$ normalized by the bubble volume fraction at the gravitational equilibrium $\left(1-\phi_{\text {grav }}\right)$ is plotted versus $I_{f}$ in Fig. 8. Here, the bubble volume fraction is favored over the liquid fraction to ease the comparison with the results obtained with granular materials. The normalization of the bubble fraction comes from the bubbles diameter and the viscosity dependence of the bubble fraction at the gravitational equilibrium. Indeed, contrary to granular materials, different foams have different bubble fractions at vanishing $I_{f}$. All the data obtained for the three different foams collapse on a single curve. The volume fraction ratio tends to one for vanishing $I_{f}$ as expected and decreases with an increase in $I_{f}$. This result proves the relevancy of the foam viscous number $I_{f}$ we propose. The inset in Fig. 8 presents the evolution of $\left(\phi_{l}-\phi_{\text {grav }}\right)$ with $I_{f}$. The asymptotic behavior of the volume fraction difference is given by the power-law $\left(\phi_{l}-\phi_{\text {grav }}\right) \propto I_{f}^{2 / 3}$. This result differs from the behavior observed in granular materials. Where the evolution is linear with the inertial number for dry media and $\sqrt{I_{v}}$ in the immersed case [10]. 


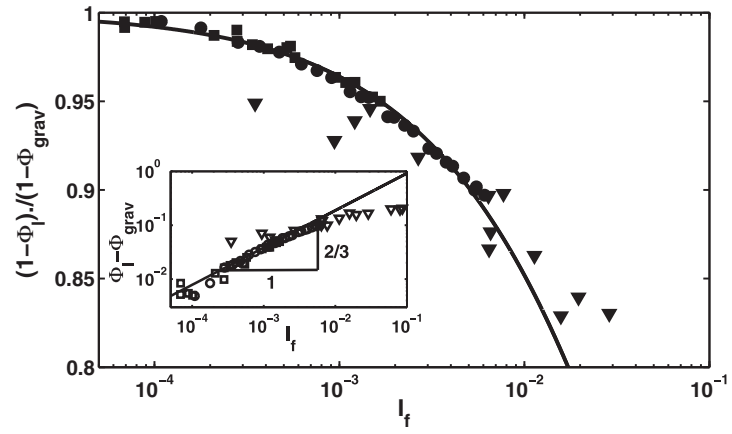

Fig. 8. Ratio between the foam mean air fraction $\left(1-\phi_{l}\right)$ and the gravitational equilibrium air fraction $\left(1-\phi_{\text {grav }}\right)$ versus the foam viscous number $I_{f}$. The inset presents the difference between the mean liquid fraction and the gravitational equilibrium liquid fraction versus the viscous number. • Case a. $\mathbf{a}$ Case b. $\mathbf{\nabla}$ Case c. The black solid line represents Eq. 6 fitted to the experimental data with $A=1.67$.

From Fig. 8, the constitutive law for the particle volume fraction $\phi=\phi(I)$ developed for granular materials seems to hold for foams. A relevant model for the evolution of the volume fraction has to respect $\left(\phi_{l}-\phi_{\text {grav }}\right) \propto I_{f}^{2 / 3}$ at vanishing $I_{f}$, to decrease and stay positive for all values of $I_{f}$ as described in [10]. A form similar to the one proposed for immersed granular materials [10] has been chosen:

$\frac{1-\phi_{l}}{1-\phi_{\text {grav }}}=\frac{\phi^{*}}{\phi^{*}+A I_{f}^{2 / 3}}$

with $A$ a free fitting parameter. This model corresponds to the black solid line in Fig. 8 with $A=1.67$ and strengthens the idea that the granular volume fraction law holds for the foams studied here as well.

The second constitutive law characterizing the granular rheology is the friction law $\tau=\mu(I) P_{p}$ [15]. An equivalent effective friction for foams might be determined from this equation. Indeed, the shear stress $\tau$ might be computed from the foam effective shear viscosity $\eta_{\text {eff }}$ rewriting Eq. 2 as $\tau=\eta_{\text {eff }} \omega$, the angular velocity being the shear rate, here. The equivalent effective friction for foams $\mu_{\text {eff }}$ is then given by the following equation:

$\mu_{\text {eff }}=\frac{\tau}{P_{p}}=\frac{\eta_{\text {eff }} \omega}{\Pi}$

and is represented in Fig. 9 as a function of the foam viscous number. Only the case $b$ is plotted in Fig. 9 since it is the only effective viscosity available. One sees that $\mu_{\text {eff }}$ is an increasing function of the viscous number. The evolution with $I_{f}$ of the curve is very similar to the effective frictions of dry/immersed granular materials $[10,11,15]$. However, at vanishing $I_{f}$, the effective friction tends to a smaller value than the typical values obtained with granular

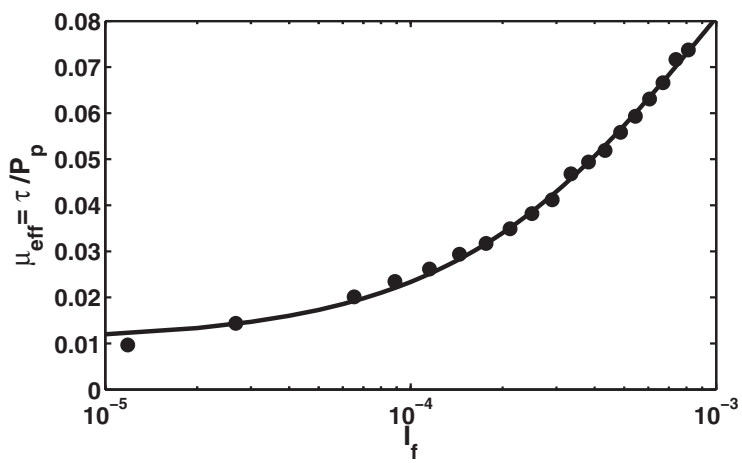

Fig. 9. Effective friction $\mu_{\text {eff }}$ as a function of the foam viscous number $I_{f}$ in semilogarithmic scale. $\bullet$ Case b. The black solid line corresponds to a fit using Eq. 8 on the experimental data. materials. Among that, within the experimental strain rate range we tested, $\mu_{\text {eff }}$ does not saturate with the viscous number but the values remains small compared to unity.

In order to complete our comparison with granular materials, the foam effective friction is modeled with the experimental friction law obtained for immersed grains in [15]. This friction law $\mu_{c}\left(I_{f}\right)$ accounts for the effects of the contact between the immersed particles and is given by the equation:

$\mu_{c}\left(I_{f}\right)=\mu_{1}+\frac{\mu_{2}-\mu_{1}}{1+I_{0} / I_{f}}$,

with $\mu_{1}, \mu_{2}$ and $I_{0}$ three free fitting parameters: $\mu_{1}$ is the minimal value of the effective friction as the viscous number $I_{f}$ vanishes; $\mu_{2}$ is the maximal value of the effective friction at large $I_{f}$. This model implies a saturation of the friction at very large $I_{f}$ (larger than the range explored in our experiments) and the fit is represented in Fig. 9 by the black solid line with the free fitting parameters $\mu_{1}=0.01, \mu_{2}=0.15$ and $I_{0}=0.001$. These values for the effective friction are consistent. The minimal value is more than one order of magnitude smaller than in the available literature on granular materials. As the contact between bubbles is softer than between granular particles, this result was to be expected. The maximal value of the effective friction is also a bit smaller (about five times) than available values for granular materials.

\section{Conclusions}

In this paper, we have compared experimental results concerning foams in a rotating drum with previous results obtained for granular materials. Foams and granular assemblies have similar flowing characteristics. Their free interface inclines during the rotation with an angle $\theta$ depending on the drum velocity $\omega$. For large $\omega$ values, the interface deforms into a S-shape with an amplitude growing with the velocity.

Given the similarities between the flows of foams and granular assemblies, the macroscopic properties of both systems have been compared. Tools developed for modeling granular frictional rheology have been applied to foams.

In order to take into account the foam properties and the role of the interstitial fluid, the foam dimensionless viscous number has been obtained by modifying the characteristic time scales proposed for granular assemblies in recent rheological descriptions. The foam behavior is governed by the competition between the two characteristic times: the rapid time of rearrangement $t_{r}$ and the mean time to move from one hole to the next one $t_{p}$. This rather simple scaling argument is shown to account for the effects of the bubble diameter and of the liquid viscosity on the foam liquid fraction.

To fully describe the macroscopic rheology of our foams, the friction law developed for immersed granular assemblies might be used. The minimal and maximal friction coefficient obtained for our foams are small compared to the values available in the granular material literature. This result was to be expected as the solid friction between bubbles is smaller than between solid particles.

This comparison between foams and immersed granular materials grasps well the foam dynamics through the foam dimensionless viscous number and through the friction constitutive law. The simple change of time scale is sufficient to take into account the flow difference between granular assemblies and foams and to describe both flows using a similar friction law.

\section{Acknowledgments}

Part of this project has been financially supported by ARC SuperCool contract of the University of Lige and by the ESA MAP Foam contract A0-108-99. AB benefits a travel grant from FRS-FNRS. 


\section{References}

[1] J. Duran, Sans, Powders, and Grains - An Introduction to the Physics of Granular Materials, Springer, New York, 2000.

[2] Y. Forterre, O. Pouliquen, Flows of dense granular media, Ann. Rev. Fluid Mech. 40 (2008) 1.

[3] GD.R. MiDi, On dense granular flows, Eur. Phys. J. E 14 (2004) 341.

[4] N.A. Pohlman, B.L. Severson, J.M. Ottino, R.M. Lueptow, Surface roughness effects in granular matter: influence on angle of repose and the absence of segregation, Phys. Rev. E 73 (2006) 031304

[5] D.V. Khakhar, A.V. Orpe, P. Andresén, J.M. Ottino, Surface flow of granular materials: model and experiments in heap formation, J. Fluid Mech. 255 (2001) 441.

[6] S.L. Pirard, G. Lumay, N. Vandewalle, J.-P. Pirard, Motion of carbon nanotubes in a rotating drum: the dynamic angle of repose and a bed behavior diagram, Chem. Eng. J. 143 (2009) 146.

[7] A.V. Orpe, D.V. Khakhar, Scaling relations for granular flow in quasi-twodimensional rotating cylinders, Phys. Rev. E 64 (2001) 031302.

[8] B. Turnbull, Scaling laws for melting ice avalanches, Phys. Rev. Lett. 107 (2011) 258001.

[9] C.C. Liao, S.S. Hsiau, K. To, Granular dynamics of a slurry in a rotating drum, Phys. Rev. E 82 (2010) 010302 (R).

[10] F. Boyer, E. Guazzali, O. Pouliquen, Unifying suspension and granular rheology, Phys. Rev. Lett. 107 (2011) 188301.

[11] B. Lecampion, D.I. Garagash, Confined flow of suspensions modelled by a frictional rheology, J. Fluid Mech. 759 (2014) 197.

[12] C. Cassar, M. Nicolas, O. Pouliquen, Submarine granular flows inclined planes, Phys. Fluids 17 (2005) 103301.

[13] I. Iordanoff, M.M. Khonsari, Granular lubrication: toward an understanding of the transition between kinetic and quasi-fluid regime, ASME J. Tribol. 126 (2004) 137.

[14] G.K. Batchelor, The effect of Brownian motion on the bulk stress in a suspension of spherical particles, J. Fluid Mech. 83 (1977) 97.

[15] C. Cassar, M. Nicolas, O. Pouliquen, Submarine granular flows inclined planes, Phys. Fluids 17 (2005) 103301.

[16] N. Vandewalle, S. Trabelsi, H. Caps, Block-to-granular transition in dense bubble flows, Europhys. Lett. 65 (3) (2004) 316.

[17] H. Caps, S. Travelsi, S. Dorbolo, N. Vandewalle, Bubble and granular flows: differences and similarities, Physica A 344 (2004) 424.

[18] R. Lespiat, S. Cohen-Addad, R. Höhler, Jamming and flow of random-closepacked spherical bubbles: an analogy with granular materials, Phys. Rev. Lett. 106 (2011) 148302.

[19] D. Weaire, W. Drenckhan, Structure and dynamics of confined foams: a review of recent progress, Adv. Colloids Interface Sci. 137 (2008) 20

[20] I. Cantat, S. Cohen-Addad, F. Elias, F. Graner, R. Höhler, O. Pitois, F. Rouyer, A Saint-Jalmes, Les Mousses: Structure et Dynamique, Belin, Paris, 2010.
[21] R. Brewster, G.S. Grest, A.J. Levine, Effects of cohesion on the surface angle and velocity profiles of granular material in a rotating drum, Phys. Rev. E 79 (2009) 011305.

[22] S.H. Chou, S.S. Hsiau, Experimental analysis of the dynamic properties of wet granular matter in a rotating drum, Powder Technol. 214 (2011) 491.

[23] N.-S. Cheng, Scaling law for velocity profiles of surface granular flows observed in rotating drum, Powder Technol. 218 (2012) 11.

[24] N. Taberlet, P. Richard, E.J. Hinch, S shape of a granular pile in a rotating drum, Phys. Rev. E 73 (2006) 050301(R).

[25] F. Pignatel, C. Asselin, L. Krieger, I.C. Christov, J.M. Ottino, R.M. Lueptow, Parameters and scalings for dry and immersed granular flowing layers in rotating tumblers, Phys. Rev. E 86 (2012) 011304.

[26] M. Maleki, M. Reyssat, F. Restagno, D. Quéré, C. Clanet, Landau-Levich menisci, J. Colloid Interface Sci. 354 (2011) 359.

[27] L. Saulnier, F. Restagno, J. Delacotte, D. Langevin, E. Rio, What is the mechanism of soap film entrainment? Langmuir 27 (2011) 13406.

[28] S. Tcholakova, D. Denkov, K. Golemanov, K.P. Ananthapadmanabhan, A. Lips, Theoretical model of viscous friction inside steadily sheared foams and concentrated emulsions, Phys. Rev. E 78 (2008) 011405.

[29] D. Denkov, S. Tcholakova, K. Golemanov, K.P. Ananthapadmanabhan, A. Lips, Viscous friction in foams and concentrated emulsions under steady shear, Phys. Rev. Lett. 100 (2008) 138301.

[30] F. Graner, B. Dollet, C. Raufaste, P. Marmottant, Discrete rearranging disordered patterns. Part I: Robust statistical tools in two or three dimensions, Eur. Phys. J. E 25 (2008) 349

[31] B. Dollet, F. Graner, Two-dimensional flow of foam around a circular obstacle: local measurements of elasticity, plasticity and flow, J. Fluid Mech. 585 (2007) 181.

[32] A. Bronfort, H. Caps, Foams in a Rotating Drum, arXiv (2014), http://arxiv.org/ abs/1406.7692

[33] P. Jop, Y. Forterre, O. Pouliquen, A constitutive law for dense granular flows, Nature 441 (2006) 727.

[34] Y. Forterre, O. Pouliquen, Flows of dense granular media, Annu. Rev. Fluid Mech. $40(2008) 1$

[35] M.L. Merrer, S. Cohen-Addad, R. Höhler, Bubble rearrangement duration in foams near the jamming point, Phys. Rev. Lett. 108 (2012) 188301.

[36] H.M. Princen, A.D. Kiss, Osmotic pressure of foams and highly concentrated emulsions. 2. Determination from the variation in volume fraction with height in an equilibrium column, Langmuir 3 (1987) 36.

[37] H.M. Princen, Highly concentrated emulsions. I. Cylindrical systems, J. Colloid Interface Sci. 71 (1979) 55

[38] H.M. Princen, Osmotic pressure of foams and highly concentrated emulsions. I. Theoretical considerations, Langmuir 2 (1986) 519.

[39] A.J. Webster, M.E. Cates, Osmotic stabilization of concentrated emulsions and foams, Langmuir 17 (2001) 595. 